\title{
Amateur Astronomers' Contribution to the HIPPARCHOS Programme
}

\author{
P. Bacchus \\ 40 rue Haute, La Grande Paroisse, F-77130 Montereau, France
}

The HIPPARCHOS satellite (High Precision Parallax Collecting Satellite) is designed to determine position, proper motion and parallax for a large number of stars. The precision expected $(0.002$ arcsec), and the absence of systematic errors with position in the sky arise from the following characteristics of the system:

- $\quad$ Measurements of angular distances between stars a long way apart $\left(58^{\circ}\right)$, are by comparison with a very stable angular reference. This reference is formed by an optical block consisting of two plane mirrors, rigidly mounted, forming an angle of $29^{\circ}$ between them, and sending two separate stellar fields into the same telescope;

- the absence of flexure (thanks to weightlessness) and of thermal deformation (non-expansion material and thermal control) ensure that the angular reference is very stable;

- $\quad$ operation outside the atmosphere allows the theoretical resolution to be reached; refraction, dispersion and atmospheric scintillation are avoided; diurnal and seasonal effects that interfere with ground-based measurements are non-existent.

The optical system is a Schmidt, with the corrector plate being replaced by figuring of the angular mirrors. The two star fields, $0.9^{\circ} \times 9^{\circ}$, are imaged on a grating consisting of 2688 slits at 1.2 arcsec intervals; rotation of the satellite causes the grating to sweep across the fields. A single star is isolated by an electronic diaphragm (dissector) for several seconds, the intensity of its light being modulated by the grating. This is analyzed by photon-counting at a sampling rate of $1200 \mathrm{~Hz}$. This give the phase of the modulation, which is equivalent to a measurement of the star's position relative to the grating. This takes place for all the programmed stars in both fields.

The catalogue of stars to be observed (the Input Catalogue) is comprehensive down to about magnitude 8 ; beyond that down to about magnitude 12 objects to be observed were drawn from suggestions made by experts in all fields of stellar astronomy. Two hundred requests for observations were made, totaling 800000 stars, but there was a lot of redundancy, leaving only 231776 different stars. From these the INCA catalogue of 114880 stars was chosen.

Many of the stars to be observed (78\%) are double or multiple. The various problems for observation and reduction that they cause are not discussed here. They also are difficult to identify, and it is here that amateurs come in. 
The Index of Double Stars (IDS), which in theory lists them in a form convenient for visual observation, is not suitable for computerized treatment, for the following reasons:

- The positions are of low accuracy $\left( \pm 0.1 \mathrm{~m}\right.$ in RA, and $\pm 1^{\prime}$ in Dec, and sometimes even more). This is not enough to set HIPPARCHOS or even an automatic transit instrument, and is not enough to find a star in a moderately rich field.

- Identifications are missing or incomplete in the major catalogues (DM, AGK3, SAO...). The IDS only gives the DM number, and sometimes omits that.

- In multiple systems, the meaning of pairs quoted (such as $A B, B C$ and $C D$ ) is often ambiguous.

- The IDS has various inconsistencies, such as giving the number of one DM star and the position of another, or else quoting positions in incompatible ways, for epochs 1900 and 2000, for example.

J. Dommanget at the Belgian Royal Observatory therefore undertook the compilation of a new catalogue of double stars, CCDM (Catalogue of Components of Double and Multiple stars), arranged by component (one line per star). Initially, the subset of CCDM stars on the INCA list has been revised, but later work will extend to all double and multiple stars. In this way we hope to obtain a catalogue with precise positions, complete identification, good internal consistency, and unambiguous identification of components. In compiling CCDM, we have to resolve thousands of problems involving insufficient or doubtful identification. It is frequently sufficient to refer to various documents (general catalogues, surveys - particularly the Palomar Sky Survey or PSS - and original references). But it is often necessary to check identification with the sky, not by measuring it, but simply by checking what there is in a field a field a few minutes across. This applies to pairs that are closer than $5^{\prime \prime}$, which are difficult to identify as double on the PSS photographs.

The work of amateurs has proved to be very valuable in identifying several hundreds of systems on the sky. In West Germany, under Dr Bastian of the RechenInstitut at Heidelberg, 20 amateurs at 8 observing stations have put in a lot of work, which, to date, has resulted in identifying 66 doubtful doubles. In doing this they have produce excellent finding charts. The exact positions of the doubles have been measured from the PSS.

In Belgium, the Royal Observatory has been helped by collaboration by several very active observers, particularly in resolving problems relating to forty-odd multiple systems incosistently described in the IDS. G. Leonis, A. Jorissen and L. Louys made three trips to ESO in Chile between 1984 and 1986, and took more than 1000 plates with the GPO ${ }^{1}$. A fourth trip, by L. Louys and J.J. Doyle, is scheduled for next August. They measured and reduced their observations with either the ORB plate-measuring machine at Brussels or ESO's at Garching. In addition, the Belgian amateurs R. Barbier, J.J. Doyle and J. Bourgeois took part in two trips to La Palma

1 The 0.4-m double astrograph, originally sited in South Africa - Eds. 
in the Canaries, where, within the framework proposed by A.N. Argue at Cambridge, they made several hundred photometric measurements of poorly-known pairs, using the 1-metre telescope and a CCD camera.

In France, amateur activity has been guided by the Double Star Section of the S.A.F. and its Director, P. Durand will describe in detail the methods used and their results. 